\title{
Rapid resolution of large mobile left ventricular thrombus with anticoagulation in dilated cardiomyopathy: A case report
}

\author{
Sunil Roy Narayanan, Nagham Saeed Jafar, Warkaa Al Shamkhani \\ Department of Cardiology, Belhoul Speciality Hospital, PO box 5527, Dubai, UAE
}

Email address:

sunilroytn@hotmail.com (S. R. Narayanan)

\section{To cite this article:}

Sunil Roy Narayanan, Nagham Saeed Jafar, Warkaa Al Shamkhani. Rapid Resolution of Large Mobile Left Ventricular Thrombus with Anticoagulation in Dilated Cardiomyopathy: A Case Report. International Journal of Medical Imaging. Vol. 2, No. 6, 2014 , pp. $156-157$. doi: 10.11648/j.ijmi.20140206.17

\begin{abstract}
Development of left ventricular thrombus is a dreaded complication after myocardial infarction and in dilated cardiomyopathy. Early detection and treatment is important as it carries the risk of systemic embolism. Thrombolytic therapy, anticoagulation and surgical removals are considered treatment options in patient with left ventricular thrombus. Resolution of left ventricular thrombus with anticoagulation demonstrate the effectiveness of such therapy in preventing the dreaded complications of clot embolism.
\end{abstract}

Keywords: Cardiomyopathy, Thrombus, Anticoagulation

\section{Case Report}

A 58 year old male presented with history of worsening exertional breathlessness, orthopnea and paroxysmal nocturnal dyspnea of two weeks duration. He had associated abdominal distension and swelling of the legs. He was diagnosed as case of dilated cardiomyopathy in 2007 when he presented with progressive heart failure symptoms and echocardiography showing global hypokinesia with severe left ventricular dysfunction. He was started on heart failure therapy including Ramipril, Carvedilol, Spironolactone and frusemide. His heart failure symptoms improved and ejection fraction improved to $45-50 \%$ and he was doing well on medical treatment for the last 6 years. His evaluation for secondary causes of cardiomyopathy could not find out any causes for cardiomyopathy and coronary angiography showed normal coronaries.

He discontinued his medications for 3 months before his presentation to emergency department with heart failure. Medicines were discontinued due to the illness and death of his wife following which he lost his job and the insurance coverage. He was consuming alcohol daily after he lost his wife. He noted worsening symptoms for few weeks but he neglected and finally decided to come to hospital when severe breathing difficulty even at rest and not able to sleep in the night. Clinically he was in severe congestive heart failure. ECG showed sinus tachycardia with left ventricular hypertrophy and strain. His Chest X-ray showed cardiomegaly with pulmonary venous Congestion. A bed side transthoracic Echo showed global hypokinesia with severe left ventricular systolic dysfunction with an LV ejection fraction of $18 \%$ and fresh large mobile thrombus in the apex of the left ventricle. (Figure A) He was started on anticoagulation with low molecular weight heparin and later overlapped with Warfarin along with reinstitution of his heart failure medications.

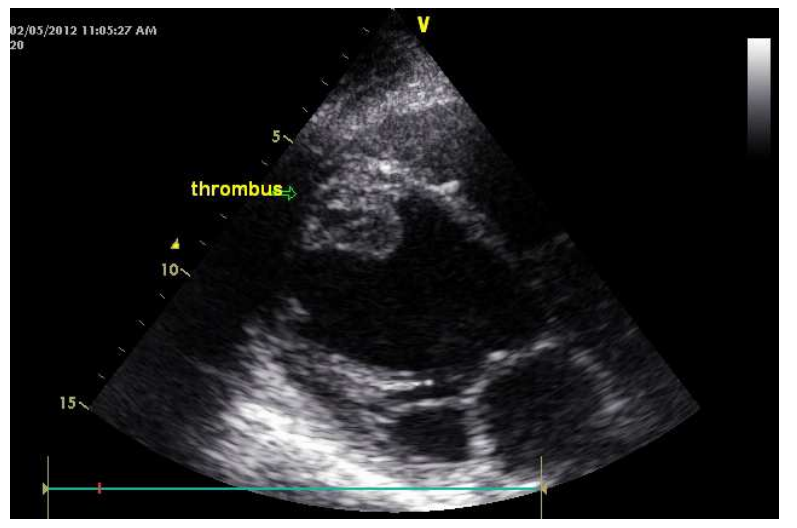

Figure A. Parasternal long axis view on TTE demonstrating multiple layered echo dense mass attached to the apex of the dilated left ventricle consistent with thrombi 
His symptoms improved following decongestive therapy. The patient condition was clinically improved and his subsequent follow up echocardiography showed resolution of the thrombus and improvement in ejection fraction. His echo done 3 months later showed left ventricular ejection fraction of $35 \%$. (Figure B, Figure C) He is doing well on medical therapy and is able to perform his activities without symptoms.

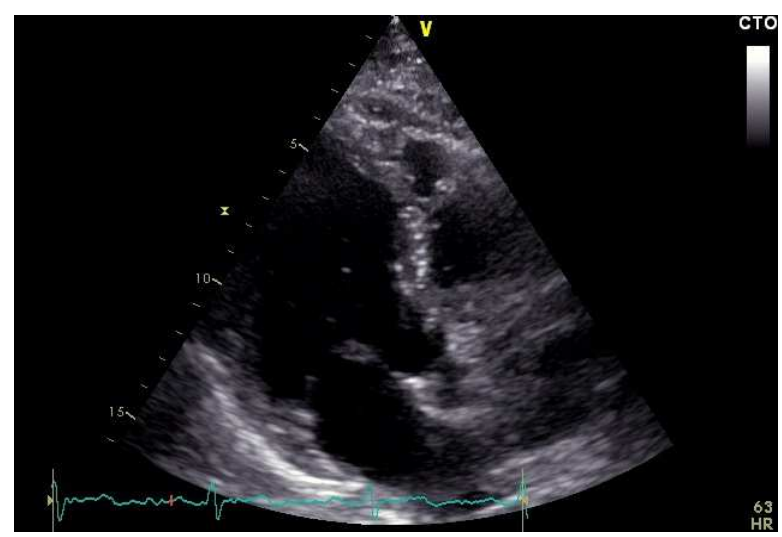

Figure B. Follow up TTE of the parasternal long axis view demonstrating significant improvement in LV dilatation and dysfunction together with complete resolution of $L V$ thrombus with anticoagulation therapy

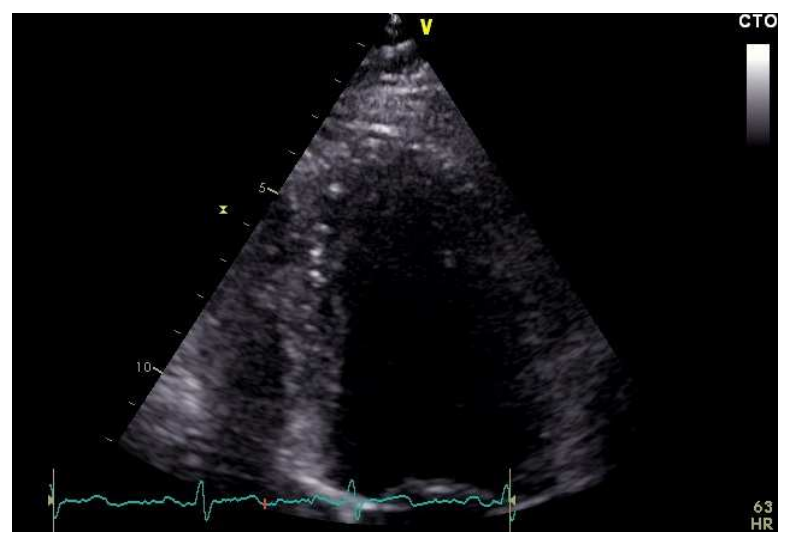

Figure C. Follow up TTE of the apical view demonstrating significant improvement in LV dilatation and dysfunction together with complete resolution of $L V$ thrombus with anticoagulation therapy.

\section{Discussion}

Early detection and treatment of left ventricular thrombus is important as it carries a high risk of thromboembolic events which can be fatal or disabling. ${ }^{1}$ Two dimensional echocardiography plays important role in the diagnosis as it has high sensitivity and specificity for detection of thrombus. Mobile or multiple thrombi and severe left ventricular dysfunction are predictors of systemic embolism. ${ }^{2}$ surgical removal of the thrombus, conventional anti-coagulation, thrombolytic therapy and antiplatelet therapy are considered the treatment options when LV thrombus is diagnosed. There is a tendency towards lower rate of embolism in the operative treatment group compared to other groups. ${ }^{3}$ However the risk of surgery is high in patient with such severe LV dysfunction and heart failure. The disappearance of thrombus with anticoagulation treatment and absence of embolic evidence suggest that when LV thrombus is diagnosed in patients with dilated cardiomyopathy or after myocardial infarction, anticoagulation may be started and surgical treatment may be delayed. In this patient with dilated cardiomyopathy, severe LV dysfunction led to the formation of mobile fresh thrombus which resolved rapidly on anticoagulation treatment.

\section{References}

[1] Pottu CR, Tulloch-Reid EE, Baugh DS et al. Left ventricular thrombus in patients with acute myocardial infarction: Case report and Caribbean focused update. Australas Med J 2012; 5: 178-83.

[2] Haugland JM, Asinger RW, Mikeli FL et al. Embolic potential of left ventricular thrombi detected by two-dimensional echocardiography. Circulation 1984;70: 588-98.

[3] Lee JM, Park JJ, Jung HW et al. Left ventricular thrombus and subsequent thromboembolism. Comparison of anticoagulation, surgical removal and antiplatelets agents. $J$ Atheroscler Thromb 2013; 20: 73-93. 